\title{
Multi-spacecraft determination of wave characteristics near the proton gyrofrequency in high-altitude cusp
}

\author{
D. Sundkvist ${ }^{1,2,3}$, A. Vaivads ${ }^{1}$, M. André ${ }^{1}$, J.-E. Wahlund ${ }^{1}$, Y. Hobara ${ }^{4}$, S. Joko ${ }^{4}$, V. V. Krasnoselskikh ${ }^{3}$, \\ Y. V. Bogdanova ${ }^{5}$, S. C. Buchert ${ }^{1}$, N. Cornilleau-Wehrlin ${ }^{6}$, A. Fazakerley ${ }^{5}$, J.-O. Hall ${ }^{2}$, H. Rème ${ }^{7}$, and G. Stenberg ${ }^{8}$ \\ ${ }^{1}$ Swedish Institute of Space Physics, Uppsala, Sweden \\ ${ }^{2}$ Department of Astronomy and Space Physics, University of Uppsala, Uppsala, Sweden \\ ${ }^{3}$ Laboratoire de Physique et Chimie de l'Environnement, CNRS, Orléans, France \\ ${ }^{4}$ Swedish Institute of Space Physics, Kiruna, Sweden \\ ${ }^{5}$ Mullard Space Science Laboratory, University College, London, UK \\ ${ }^{6}$ Centre d'Etude des Environnements Terrestre et Planetaires, CNRS, Vélizy, France \\ ${ }^{7}$ Centre d'Etude Spatiale des Rayonnements, CNRS, Toulouse, France \\ ${ }^{8}$ Department of Physics, University of Umeå, Umeå, Sweden
}

Received: 30 September 2004 - Revised: 28 January 2005 - Accepted: 2 February 2005 - Published: 30 March 2005

\begin{abstract}
We present a detailed study of waves with frequencies near the proton gyrofrequency in the high-altitude cusp for northward IMF as observed by the Cluster spacecraft. Waves in this regime can be important for energization of ions and electrons and for energy transfer between different plasma populations. These waves are present in the entire cusp with the highest amplitudes being associated with localized regions of downward precipitating ions, most probably originating from the reconnection site at the magnetopause. The Poynting flux carried by these waves is downward/upward at frequencies below/above the proton gyrofrequency, which is consistent with the waves being generated near the local proton gyrofrequency in an extended region along the flux tube. We suggest that the waves can be generated by the precipitating ions that show shell-like distributions. There is no clear polarization of the perpendicular wave components with respect to the background magnetic field, while the waves are polarized in a parallelperpendicular plane. The coherence length is of the order of one ion-gyroradius in the direction perpendicular to the ambient magnetic field and a few times larger or more in the parallel direction. The perpendicular phase velocity was found to be of the order of $100 \mathrm{~km} / \mathrm{s}$, an order of magnitude lower than the local Alfvén speed. The perpendicular wavelength is of the order of a few proton gyroradius or less. Based on our multi-spacecraft observations we conclude that the waves cannot be ion-whistlers, while we suggest that the waves can belong to the kinetic Alfvén branch below the proton gyrofrequency $f_{c p}$ and be described as non-potential ion-cyclotron waves (electromagnetic ion-Bernstein waves) above. Linear
\end{abstract}

Correspondence to: D. Sundkvist

(davids@irfu.se) wave growth calculations using kinetic code show considerable wave growth of non-potential ion cyclotron waves at wavelengths agreeing with observations. Inhomogeneities in the plasma on the order of the ion-gyroradius suggests that inhomogeneous (drift) or nonlinear effects or both of these should be taken into account.

Keywords. Magnetospheric physics (magnetopause, cusp and boundary layers; plasma waves and instabilities) - Space plasma physics (waves and instabilities)

\section{Introduction}

The cusp regions of the terrestrial magnetosphere play an important role in the transfer of energy from the solar wind to the ionosphere, since the cusp magnetic field lines directly connect the solar wind with the Earth's polar regions. The cusps are also regions where efficient energization of the ionospheric plasma is taking place and energy is continuously transferred among different plasma populations. Most of these processes are due to different plasma waves.

Intense waves with frequencies of the order of the ion gyrofrequency have often been observed in the cusp by several spacecraft at varying altitudes. Such waves are known to be important for energy redistribution between different particle populations, e.g. via ion-cyclotron resonance. Identification of the wave generation mechanisms and wave modes are essential for the understanding of the overall cusp energy conversion processes and particle transport.

The cusp is a very structured region. Until recently only single satellite observations have been available, with the inherent problem of distinguishing between spatial and 
temporal variations. Here we analyze wave observations obtained at around 8 Earth radii $\left(R_{E}\right)$ near noon by the four Cluster spacecraft. We find that multi-spacecraft measurements are essential for understanding wave properties in the cusp.

Waves with obvious peaks in the power spectrum near the proton gyrofrequency have been observed in the auroral and cusp regions, see reviews by Gurnett (1991) and André (1997). They have been identified either as Electrostatic Ion Cyclotron (EIC) waves, typically with a spectral peak above the proton gyrofrequency (e.g. Kaufmann and Kintner, 1982; André et al., 1987; Stenberg et al., 2002), or as Electromagnetic Ion Cyclotron (EMIC) waves, usually with a peak below the proton gyrofrequency but sometimes also above (e.g. Temerin and Lysak, 1984; Gustafsson et al., 1990; Erlandson and Zanetti, 1998; Chaston et al., 1998, 2002; Santolík et al., 2002). Possible generation mechanisms include particle beams along the geomagnetic field. There are several reports of EMIC specifically in the cusp (Russel et al., 1971; Scarf et al., 1972; Fredricks and Russell, 1973).

Other commonly observed types of waves have broadband spectra, covering frequencies from below one up to at least several hundred Hz. These spectra often have no clear signature near the ion gyrofrequency (e.g. Gurnett and Frank, 1977; Kintner et al., 2000; Wahlund et al., 2003) and are often associated with local ion energization (e.g. André et al., 1998; Hamrin et al., 2002; Bouhram et al., 2003a, 2003b). Several studies with Interball-1 (see Savin et al., 2004, and references therein) focus on the boundary between cusp and magnetosheath. In a region identified as the turbulent boundary layer in the distant cusp broadband turbulent like spectra are often observed. The generation mechanism of these waves and their dispersive properties are still not clear.

Recent studies using the Polar (Le et al., 2001) and Cluster (Nykyri et al., 2003, 2004) spacecraft study waves in the cusp having clear spectral peaks at frequencies near the proton gyrofrequency. These waves were present in the cusp itself, as well as in its equator- and poleward boundaries. The average Poynting flux was found by Le et al. to be mainly earthward and the ratio of the wave electric and magnetic field amplitudes was close to the local Alfvén speed. Both Nykyri et al. and Le et al. found that the waves could have both left- and right-handed polarization and different angles with respect to the ambient magnetic field. In addition, a time-domain cross correlation analysis done by Nykyri et al. showed that there is no correlation of the signals between spacecraft with separation distances as small as $\sim 100 \mathrm{~km}$. The free energy source and generation mechanism of the waves is not clear, even though Nykyri et al. speculates that it can be due to velocity shear present in the cusp.

In our study we analyze waves in the frequency range around the proton gyrofrequency as observed in the highaltitude cusp by the Cluster spacecraft. We particularly concentrate on the poleward boundary of the cusp that for northward Interplanetary Magnetic Field (IMF) conditions corresponds to the region of freshly reconnected (opened) field lines. The goal of our investigation is to describe the polarization and dispersive properties of the waves in enough detail to understand their nature (wave mode identification) and to identify the free energy source that could generate the waves.

This paper uses data from the multi-spacecraft Cluster encounter of the high-altitude cusp on 9 March 2002. We start the observations section (2) with a general overview of the cusp crossing from a particle- and wave point of view. We then discuss measurements of the Poynting- and ion power flux and carry out a polarization analysis. Then we move on to multi-spacecraft measurements and present the results from a multi-spacecraft cross-spectral analysis. Finally, we end the observations part by showing ion distribution functions. The discussion of the measurements in Sect. 3 is focused on wave properties, wave identification and possible wave generation mechanisms.

\section{Observations}

\subsection{Event overview}

On 9 March 2002 the Cluster spacecraft crossed the highaltitude cusp of the Northern Hemisphere. The Cluster spacecraft were at the short separation of $\sim 100 \mathrm{~km}$. All observations in this section are presented for spacecraft number four (C4), although the other spacecraft observe the same characteristic features. Figure 1 shows an overview of the crossing. The Cluster spacecraft exit the polar cap and enter the boundary layer between the polar cap and the cusp at $(1.6,-1.2,7.6) \mathrm{GSM}$ around $02: 36 \mathrm{UT}$, enter the high latitude boundary layer (HLBL) at $\sim 02: 49$ UT, and finally enter the cusp proper at 03:02 UT (borders are marked by dotted lines in Fig. 1).

The regions are easiest to identify from Figs. 1a, b, c that show proton number flux, proton pitch angle distribution and proton density (CIS experiment, Rème et al., 1997). The boundary layer between polar cap and cusp is characterized by the apperance of solar wind particles while their density is still very low. The cusp, consisting of both HLBL and the proper cusp, can be clearly identified as a region of high plasma density, $n>1 \mathrm{~cm}^{-3}$. The HLBL can be distinguished by the presence of intense ion injections. In the cusp proper both up- and downgoing ions are observed, as seen from the pitch angle distribution in Fig. 1, panel (b).

The interplanetary magnetic field (ACE data time lagged by $60 \mathrm{~min}$ ), corresponding to the Cluster cusp crossing at 02:49 UT, was $(-3,4,2)$ nT (GSM). For these IMF conditions the expected reconnection site at the magnetopause is on the duskside, tailward from the cusp. This is consistent with the precipitating energetic ions observed by Cluster between 02:49-03:02 UT being reconnection jets generated at newly-reconnected field lines. For southward IMF and low latitudes this type of plasma would be denoted as cleft or as the low-latitude boundary layer (LLBL); here observed for northward IMF we refer to it as a high-latitude boundary layer (HLBL). 
Figure 1, panel (d) shows a pitch angle distribution of the electron flux centered around $130 \mathrm{eV}$ (the PEACE instrument, Johnstone et al., 1997). This energy corresponds approximately to the energy at which cusp electrons have a maximum flux and all essential electron features can be identified in this panel. A significant increase in electron flux is observed at the same time as the increased proton flux around 02:49 UT. Also, one can see that there is a lower flux of electrons in the HLBL than in the proper cusp. In both regions the pitch-angle distribution show that the electrons are anisotropic, with the highest fluxes being along the ambient magnetic field.

The ambient magnetic field (FGM experiment, Balogh et al., 1997) during this event was of the order of 105-110 nT, and is displayed in panel (e). A continuous decrease of the magnetic field amplitude is due to the spacecraft motion to lower magnetic latitude and higher altitude. No clear decrease in the magnetic field magnitude is associated with the cusp entrance, as is sometimes observed in the high-altitude or exterior cusp.

The above identified different regions (polar cap, boundary layer between polar cap and cusp, HLBL, proper cusp) also show up clearly in the plasma wave emissions. Enhanced broad-band electric field emissions, (EFW experiment, Gustafsson et al., 1997) are observed in the boundary layer between the polar cap and cusp, as well as in the HLBL but diminishes before entering the proper cusp, shown in panel (f). Also, the magnetic field shows broadband emissions, panel (g) (STAFF experiment, CornilleauWehrlin et al., 1997), extending throughout the proton gyrofrequency $f_{c p} \approx 1.6 \mathrm{~Hz}$ and having the highest amplitudes inside the cusp; the emissions are weaker in the cusp proper than in the HLBL. These emissions are the subject of this paper and in the following sections we will analyze them in detail.

\subsection{Wave occurrence and general characteristics}

Panel (f) in Fig. 1 shows an electric field wavelet spectrogram. Broad-band, low-frequency emissions are observed to occur from 02:36 UT when Cluster was exiting the polar cap until the end of the interval, with diminishing power from around 03:00 UT when entering the cusp proper. Note that the electric field wave power is roughly constant during 02:36-03:00 UT. No clear sign of entering the HLBL at $\sim 02: 49$ UT can be noticed in the $\boldsymbol{E}$-field spectrogram. Different features compared to the electric field can be noted in the magnetic field wavelet spectrogram, panel $(\mathrm{g})$ of Fig. 1. Strong wave emissions in the magnetic field begin at $\sim 02: 49$ UT and seem to be directly related to higher density and particle flux in the HLBL and cusp proper. The strong emission near $0.5 \mathrm{~Hz}$ in the beginning of the interval is due to a residual overtone of the spacecraft spin frequency. Between 02:50-03:02 UT the intensity of magnetic field emissions stays roughly constant, except for two features that can be noted in this time interval. At 02:54 and 02:58 UT Cluster observes depressed magnetic field wave power, where CIS observes lower density and PEACE observes almost no electron flux. In the cusp proper, from 03:02 UT onwards, there are magnetic field emissions but their intensity is less than in the HLBL.

\subsection{Poynting and ion power flux}

To analyze the energy transport during the event we calculate the total integrated Poynting flux of the waves, the distribution of Poynting flux over frequencies and the ion power flux. The calculations are carried out in the spacecraft frame of reference and the results are presented in Fig. 1. Panel (h) shows a wavelet spectrogram of the Poynting flux along the ambient magnetic field, calculated from the EFW and STAFF instruments. The EFW instrument measures only two components of the electric field, i.e. the electric field of the spin plane of the spacecraft, while STAFF measures the full three-dimensional magnetic field. The Poynting flux along the background magnetic field can still be calculated without any further assumptions, since the deviation of the spin plane normal from the background magnetic field vector is practically negligible for this event. We note that inside the cusp there is a relatively sharp boundary at about the proton gyrofrequency $f_{c p} \approx(1.6 \mathrm{~Hz})$, such that the Poynting flux of the waves with lower frequencies is predominantly parallel to the background magnetic field (red) while the Poynting flux of the waves with higher frequencies is mainly antiparallel (blue). Since this is a Northern Hemisphere cusp crossing, parallel in this case means earthward (downward). The sharp boundary near the proton gyrofrequency indicates that there probably is no important Doppler shift in frequency. An exception is the period between 02:5602:58 UT, where waves within the whole frequency range $0.3-10 \mathrm{~Hz}$ are mostly downgoing. This time period is also associated with a fast $(\approx 150 \mathrm{~km} / \mathrm{s})$ field-aligned plasma flow. The flow can cause a Doppler shift that could be one possible explanation why only downgoing Poynting flux is detected. The fact that we generally observe Poynting flux in different directions, depending on frequency, is consistent with locally generated waves (see Discussion, Sect. 3).

Panel (j) in Fig. 1 shows the time integral of the fieldaligned component of the ion power flux (IPF, black line) and the Poynting flux (colored lines). The three colored lines correspond to three different frequency intervals: red $(0.01-$ $0.1 \mathrm{~Hz})$, green $(0.3-1.6 \mathrm{~Hz})$ and blue $(1.6-10 \mathrm{~Hz})$. The green and blue lines have been multiplied by 2 and 40, respectively, for comparison purposes. The low-frequency $(0.01-$ $0.1 \mathrm{~Hz}$ ) Poynting flux (red line) has been calculated by using the EFW and FGM instruments, while the flux in frequency range $0.3-10 \mathrm{~Hz}$ uses the EFW and STAFF instruments. This division of frequencies is due to the spacecraft spinfrequency $(0.25 \mathrm{~Hz})$, and $f_{c p}(1.6 \mathrm{~Hz})$. A positive/negative trend corresponds to mostly downgoing/upgoing flux. We note that most Poynting flux is in the lowest frequencies, and is predominantly downgoing. These waves have periods of about $10 \mathrm{~s}$ to $1.5 \mathrm{~min}$ and are probably low-frequency Alfvén waves. They start to show significant downward Poynting 


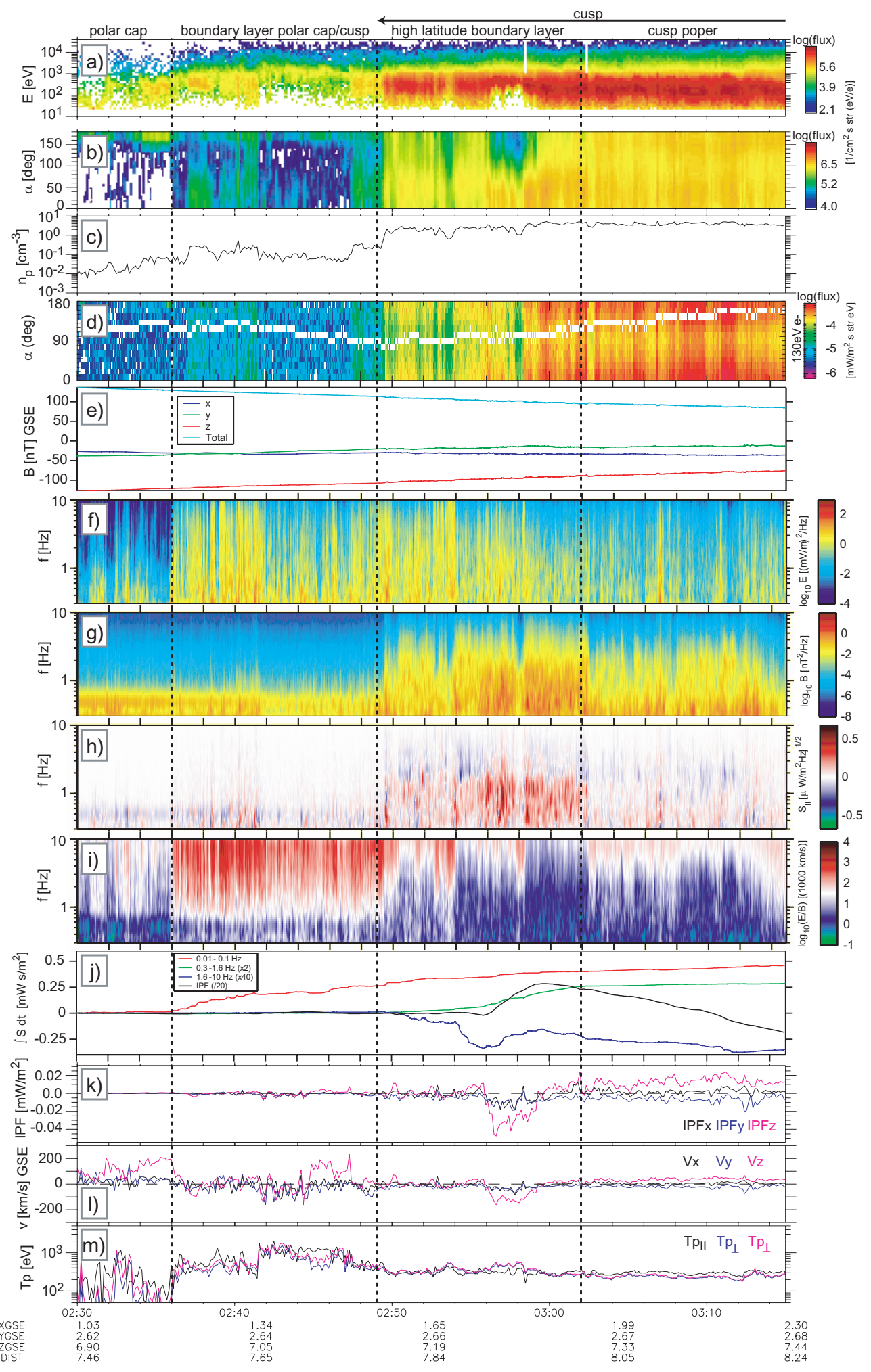

Fig. 1. Northern outbound cusp crossing by Cluster spacecraft 4 at 9 March 2002. The panels from top to bottom are (a) proton number flux, (b) proton pitch angle distribution, (c) proton density from CIS, (d) electron pitch angle distribution, (e) magnetic field from FGM, (f) spectrogram of the electric field, (g) spectrogram of the magnetic field, (h) Poynting flux along the ambient magnetic field, (i) $\delta E_{\perp} / \delta B_{\perp}-$ ratio, (j) integrated parallel Poynting flux and ion power flux, (k) ion power flux, (l) velocity moment, (m) proton temperature. The dotted lines mark the boundaries of the different regions that are identified and described in the text. 
flux inside the boundary layer between the polar cap and the cusp, and continue to do so in the HLBL and cusp proper without any significant changes in features. The total wave Poynting flux in the interval $0.3-1.6 \mathrm{~Hz}$ is downgoing and is only observed in the HLBL, where it is comparable in magnitude to the lower frequency Poynting flux. Above $(1.6 \mathrm{~Hz})$ the flux is much smaller in magnitude and mainly upward, except for 02:56-02:59 UT where it is downward, corresponding to the interval of enhanced proton injection. It is important to note that the field-aligned ion power flux is $\sim 40$ times larger than the field-aligned Poynting flux of waves with frequencies $0.3-10 \mathrm{~Hz}$.

\subsection{Wave polarization}

Panel (i) of Fig. 1 shows the ratio of the perpendicular electric and magnetic wave fields, the $\delta E_{\perp} / \delta B_{\perp}$-ratio. The value of zero in the colorbar corresponds to $1000 \mathrm{~km} / \mathrm{s}$. This can be compared to the Alfvén speed, which is of the order of $1300-1500 \mathrm{~km} / \mathrm{s}$ in the HLBL, and $800-900 \mathrm{~km} / \mathrm{s}$ in the cusp proper. It can be seen that the $\delta E_{\perp} / \delta B_{\perp}$-ratio inside the cusp for frequencies below the proton gyrofrequency is close to the Alfvén velocity while it increases with higher frequencies. This can be clearly seen in Fig. 2, where we show a line spectra of the $\delta E_{\perp} / \delta B_{\perp}$-ratio calculated for a time interval in the HLBL. Panel (a) shows the power spectral density of the wave electric field $\delta E$ from $\mathrm{C} 4$, from the time interval 02:54:00-02:56:00 UT (blue line), and the corresponding power spectral density of the magnetic field $\delta B$ from STAFF (red line). Panel (b) shows the ratio $\delta E_{\perp} / \delta B_{\perp}$. The ratio stays constant when $f \rightarrow 0$ and is of the order of the local Alfvén speed.

The polarization parameters for the magnetic field, as defined by Carozzi et al. (2001) were calculated in a fieldaligned coordinate system and are presented for $\mathrm{C} 4$ in Fig. 3 . We have chosen to present the polarization analysis in a fieldaligned coordinate system rather than a minimum variance frame, since this representation is closer to a theoretical description of possible wave modes. In a field-aligned coordinate system right or left handness can be inferred directly by calculating the polarization parameters of the perpendicular components. Figure 3a shows the power spectral density of the magnetic field as measured by FGM. The degree of polarization of the two magnetic field components perpendicular to the ambient magnetic field is shown in panel (b) while in panel (c), the ellipticity is shown for the same components. The degree of polarization is low and no definite polarization in terms of right- or left handedness can be seen in the perpendicular plane.

If the same polarization analysis is carried out in the plane spanned by the ambient magnetic field direction and the sunward pointing perpendicular direction, a clear polarization is observed. For this case Fig. 3, panel (d), shows a higher degree of polarization for frequencies below and slightly above $f_{c p}$. The proton gyrofrequency is clearly visible in panel (e), where the ellipticity changes sign from positive to negative, meaning that the sense of polarization changes direction in

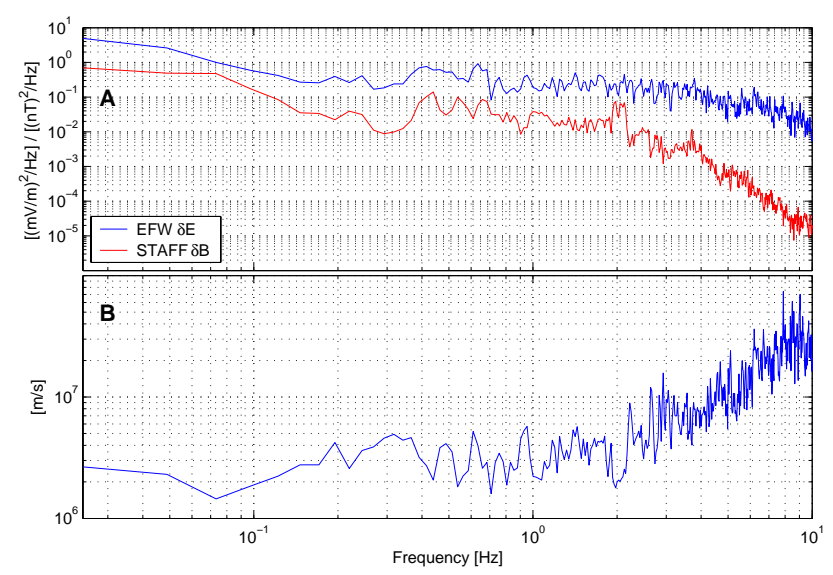

Fig. 2. Panel (a) Power spectral density of the electric and magnetic field and panel (b) the $\delta E_{\perp} / \delta B_{\perp}$-ratio for the time interval 02:54:00-02:56:00 UT in the HLBL. In the low frequency limit the $\delta E_{\perp} / \delta B_{\perp}$-ratio is of the order of the Alfvén velocity (1300$1500 \mathrm{~km} / \mathrm{s}$ ). The proton gyrofrequency was $\sim 1.6 \mathrm{~Hz}$.

this plane. If the sunward pointing perpendicular component is exchanged for the duskward perpendicular component, a higher degree of polarization is again observed, panel (f), though the ellipticity signature is not as evident in this case, panel $(\mathrm{g})$. This feature of the polarization is consistent with a change in sign of one component of $\boldsymbol{k}$, i.e. either $k_{\perp}$ or $k_{\|}$ (see Discussion).

Examining hodograms of the magnetic field, Fig. 4, corresponding to the projections used in Fig. 3, reveals the reason for the degree of polarization found. Figure 4a shows a hodogram representation of the perpendicular components from the wave magnetic field of $\mathrm{C} 4$, band-pass filtered between $1.0-1.5 \mathrm{~Hz}$ (below $f_{c p}$ ). It is noticed that the polarization changes direction on a time scale compared to the wave period. The time scale for change in the sense of polarzation is shorter than the time scale over which averaging is made for the spectrograms in Fig. 3, hence the low degree of polarization in this projection plane. Figures $4 \mathrm{~b}$ and $4 \mathrm{c}$ shows hodogram representations for the two perpendicular vs. parallel projections. In this case the period for polarization changes is longer, resulting in a higher degree of polarization. The same kind of hodograms but now band-pass filtered at $1.8-2.3 \mathrm{~Hz}$ (above $f_{c p}$ ) are presented in panels (d), (e) and (f). The same features are seen in this case, except that the sense of polarity has changed, panel (e).

\subsection{Wave multi-spacecraft analysis}

The short separation of the spacecraft allows for the utilization of multi-spacecraft techniques to gain information on wave properties. Figure 5 shows the configuration of the spacecraft with respect to the ambient magnetic field at 03:00 UT. C1 and C2 are aligned almost along the same field line. Their perpendicular separation was only $29 \mathrm{~km}$, while their parallel separation was $76 \mathrm{~km}$. No other pair of spacecraft was located so close to each other in the 

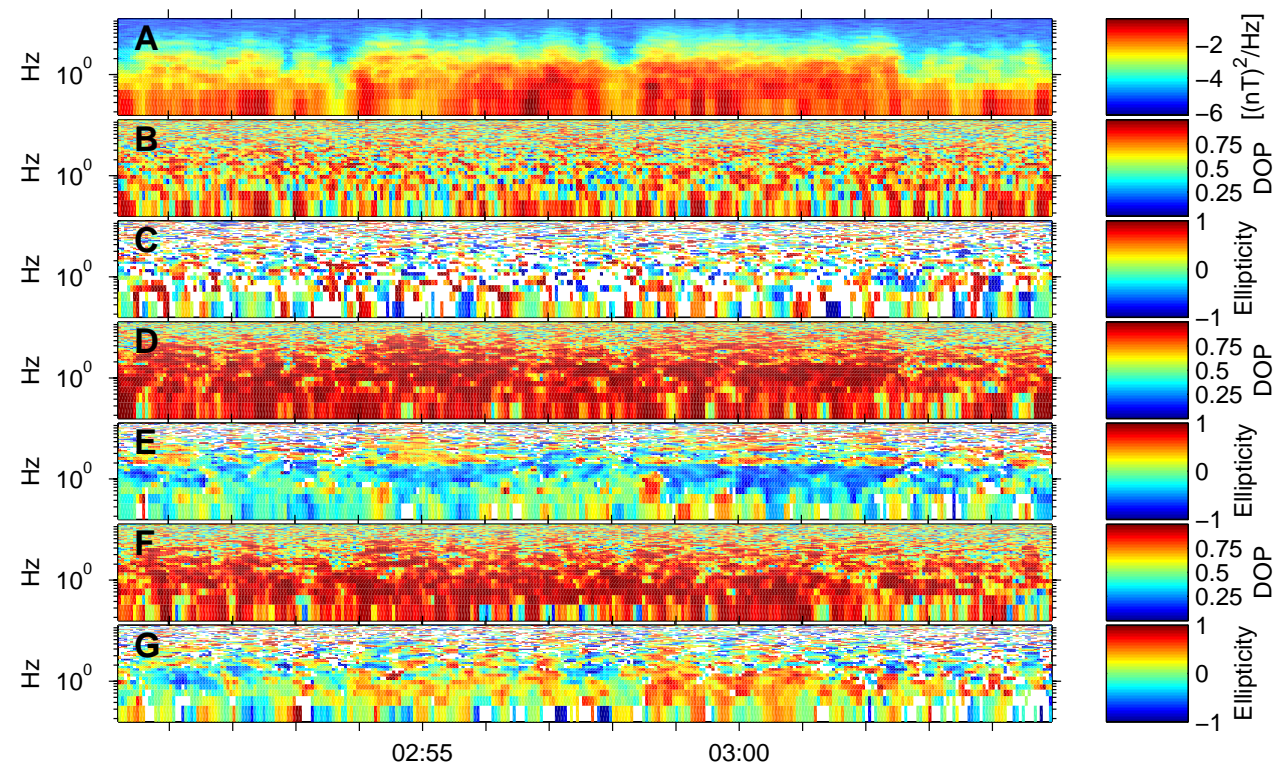

Fig. 3. Polarization in a field-aligned coordinate system as a function of frequency. Panel (a) shows the Fourier transform spectrogram of the FGM magnetic field. Panel (b) shows the degree of polarization and panel (c) the ellipticity for the perpendicular components. Similarly for the sunward-pointing perpendicular and the parallel components in panels (d) and (e). Finally, panels (f) and (g) shows the same parameters for the duskward-pointing perpendicular and the parallel components.
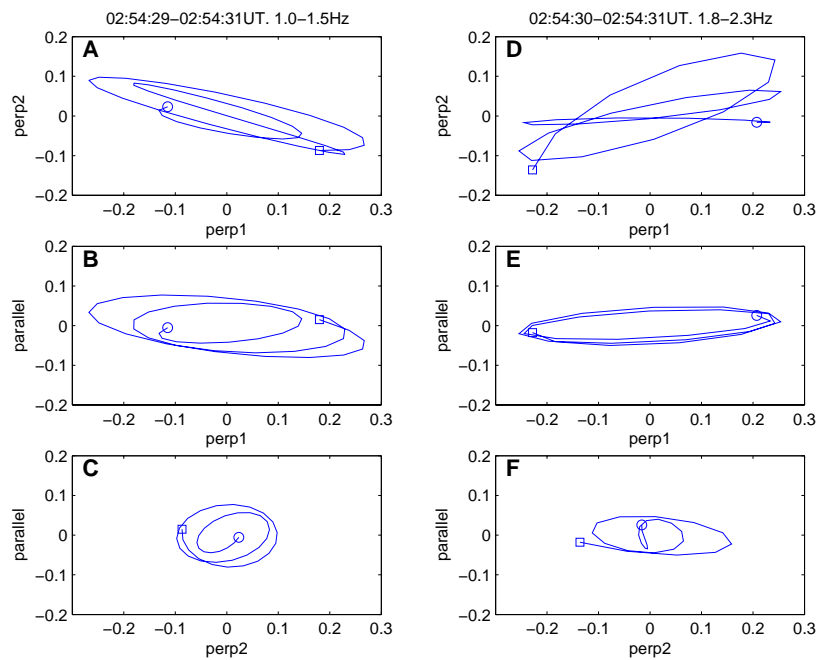

Fig. 4. Hodograms of the wave magnetic field from $\mathrm{C} 4$ in a fieldaligned coordinate system, filtered in two different frequency intervals below and above $f_{c p}$, respectively. The square marks the start of the time series, and the circle the end.

perpendicular direction. On the other hand, e.g. the pair $\mathrm{C} 1$ C4 was separated $43 \mathrm{~km}$ in parallel direction, roughly half the parallel distance of the pair $\mathrm{C} 1-\mathrm{C} 2$, and $63 \mathrm{~km}$ in the perpendicular direction. For comparison, the proton gyroradius was approximately $\rho_{p} \sim 23 \mathrm{~km}$ (calculated for a mean energy of $300 \mathrm{eV}$, see Fig. 1a).

We show the results of a cross-spectral analysis using the sunward pointing perpendicular component of the magnetic field. Similar results are obtained using the other components. The cross-spectrum of two time signals, $s_{i}$ and $s_{j}$, is defined by (e.g. Labelle and Kintner, 1989)

$C_{i j}(\omega)=\frac{\left\langle S_{i} S_{j}^{*}\right\rangle}{\sqrt{\left\langle\left|S_{i}\right|^{2}\right\rangle\left\langle\left|S_{j}\right|^{2}\right\rangle}}=\gamma(\omega)^{2} e^{i \Delta \theta_{i j}(\omega)}$,

where $S_{i}$ and $S_{j}$ are the Fourier transform of $s_{i}$ and $s_{j}$, respectively, and angle brackets represent ensamble averages. In practice, the ensamble average is realized by taking time averages with a window of suitable length, due to the inherent problem of observing the same physical process with repeated observations in satellite measurements. The amplitude $\gamma^{2}$ denotes the coherency of the cross-spectrum, while $\Delta \theta_{i j}$ is the phase.

Figure 6 shows an example of a cross-spectral analysis of signals from the pairs $\mathrm{C} 1-\mathrm{C} 2$ and $\mathrm{C} 1-\mathrm{C} 4$, respectively; the examples are from HLBL region. The coherency, Fig. 6b, is high in a large frequency band centered around $f_{c p}$ for the pair C1-C2 (red line). In panel (c) the phase difference between the signals is plotted as a function of frequency. There is a clear trend in phase difference in a wide frequency range around $f_{c p}$ (red crosses). This indicates that these waves most probably satisfy the same dispersion relation and in addition, it can be seen that $2 \pi$ wrapping is not involved. The relation between phase and frequency in the frequency interval around $f_{c p}$ results in a phase velocity projected on the spacecraft separation axis $\boldsymbol{d}_{\boldsymbol{i} \boldsymbol{j}}$. For a plane wave the phase difference between two spatially separated points (two spacecraft) is given by

$\Delta \theta_{i j}=\theta_{i}-\theta_{j}=\boldsymbol{k} \cdot \boldsymbol{d}_{\boldsymbol{i} \boldsymbol{j}}$ 


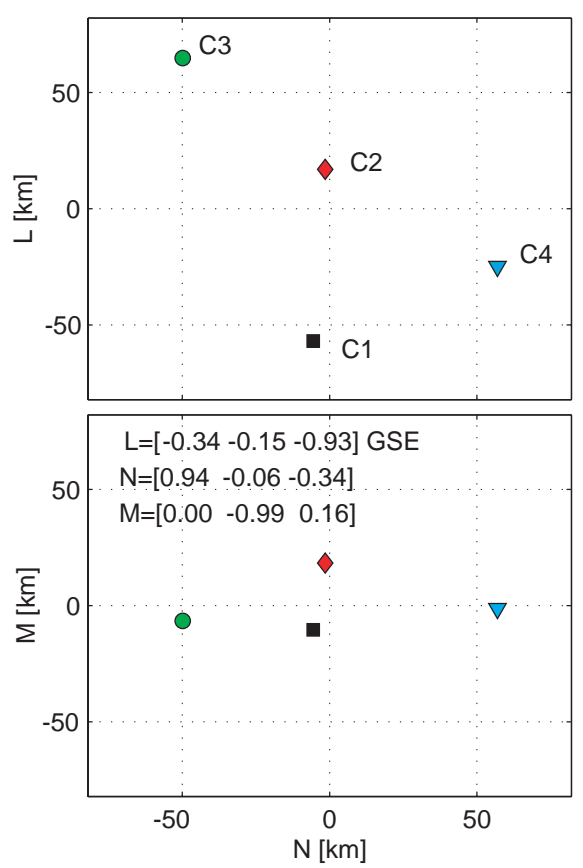

Fig. 5. Location of the Cluster spacecraft corresponding to Fig. 6. $\mathrm{L}, \mathrm{M}$ and $\mathrm{N}$ are the parallel and two perpendicular axes, respectively, in a field-aligned coordinate system. $\mathrm{L}$ is the field-aligned direction, $\mathrm{N}$ is the perpendicular component pointing towards the Sun, and $\mathrm{M}$ completes the right-handed system. At this time $\mathrm{C} 1$ and $\mathrm{C} 2$ are at the smallest perpendicular separation and hence almost on the same field line.

where $\boldsymbol{k} \cdot \boldsymbol{d}_{i j}=k_{i j} d_{i j}$ is the projection of the k-vector on the interferometer axis. This gives the phase velocity along the interferometer axis

$v_{p h, i j}=\frac{2 \pi f}{k_{i j}}=\frac{2 \pi f}{\Delta \theta_{i j} / d_{i j}}$.

Here $\Delta \theta_{i j}$ in Eqs. (2), (3) is the same as in Eq. (1). Using values from Fig. 5 and Fig. 6 results in a projected phase velocity of approximately $v_{p h,(C 1-C 2)} \sim 250-300 \mathrm{~km} / \mathrm{s}$. Since $k_{i j} \leq k$ always holds true, the phase velocity estimate along the interferometry axis is an upper bound on the true phase velocity, i.e. $v_{p h} \leq v_{p h, i j}$.

The separation axis is mostly along the parallel direction, which, at first sight, seems to imply that the obtained value of the projected phase velocity is a good estimate of a parallel phase velocity. However, this is true only if the phase velocity of the wave is almost parallel with respect to the ambient magnetic field, i.e. $k_{\|}>k_{\perp}$. If the phase velocity is almost perpendicular, $k_{\perp}>k_{\|}$, then even though the separation axis is close to being parallel with respect to the ambient magnetic field, the parallel phase velocity and phase velocity along the separation axes can differ significantly. In this case one can still use the phase velocity along the separation axis to estimate the perpendicular phase velocity. There is additional information that allows us to speculate that the waves in our case satisfy the condition $k_{\perp} \gg k_{\|}$.
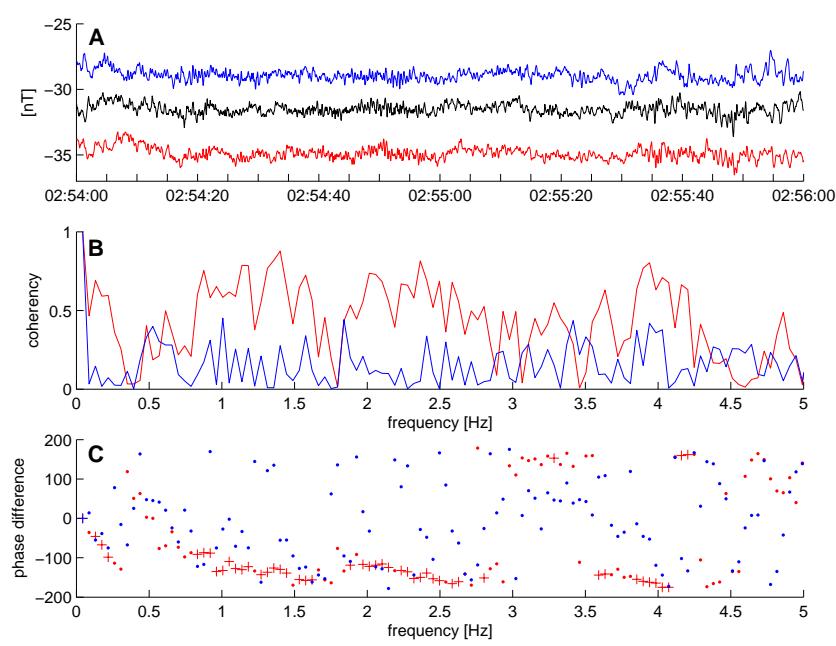

Fig. 6. Cross spectral analysis of the sunward-pointing perpendicular magnetic field component for the spacecraft pairs $\mathrm{C} 1-\mathrm{C} 2$ which were situated almost along the same field line, and $\mathrm{C} 1-\mathrm{C} 4$ which had a larger perpendicular separation but a closer parallel separation. (a) Time series of the magnetic field. Black, red and blue are C1, C2 and $\mathrm{C} 4$, respectively. $\mathrm{C} 2$ and $\mathrm{C} 4$ have been shifted by $-3 \mathrm{nT}$ and $+3 \mathrm{nT}$, respectively, for clarity. (b) Coherency from cross-spectral analysis. Red from the pair C1-C2, and blue from C1-C4. (c) Phase difference between the signals. The different markers corresponds to points with $\gamma^{2} \geq 0.5$ (crosses) or $\gamma^{2}<0.5$ (dots). Colors as in panel (b).

In Sect. 2.3, Fig. 1c, we showed that the Poynting flux changes direction at $f_{c p}$, which for wave modes where the parallel group velocity is in the same direction as the parallel phase velocity implies that $k_{\|}$should also change sign at $f_{c p}$. This is not observed in Fig. 6c. The slope is about the same above and below $f_{c p}$, although a jump in the slope is observed. This is consistent with the Poynting flux results, if $k_{\perp} \gg k_{\|}$holds true. The measured phase difference will then be an estimate of a perpendicular phase velocity, since $\boldsymbol{k} \cdot \boldsymbol{d}=\left(\boldsymbol{k}_{\perp}+\boldsymbol{k}_{\|}\right) \cdot \boldsymbol{d} \approx \boldsymbol{k}_{\perp} \cdot \boldsymbol{d}$. The effect of $k_{\|}$changing sign at $f_{c p}$ will still be notable as a discontinuous jump in the phase difference versus frequency diagram, as is observed in Fig. 6c. Since the perpendicular separation is about 2-3 times smaller compared to $d_{12}, v_{p h,(C 1-C 2)}$ will be an upper bound, and the final estimate of $v_{p h, \perp}$ is of the order of $v_{p h, \perp} \sim 100 \mathrm{~km} / \mathrm{s}$. We note that this phase velocity has been calculated in the spacecraft frame of reference, and that a possible Doppler shift could alter the value in the plasma rest frame. This phase velocity is much smaller than the Alfvén velocity. Small phase velocities are observed regardless of whether the spacecraft are located in the HLBL or the cusp proper in this event. The phase velocity in the frequency interval $1-2 \mathrm{~Hz}$ also gives an upper bound estimate of the perpendicular wavelength $\lambda_{\perp} \sim 50-100 \mathrm{~km}$.

The same kind of analysis is presented for the pair $\mathrm{C} 1-\mathrm{C} 4$ with blue lines and markers in Fig. 6. One of the spacecraft $(\mathrm{C} 1)$ is the same in these two pairs. As is evident from panel (b) there is almost no coherency between the signals of 

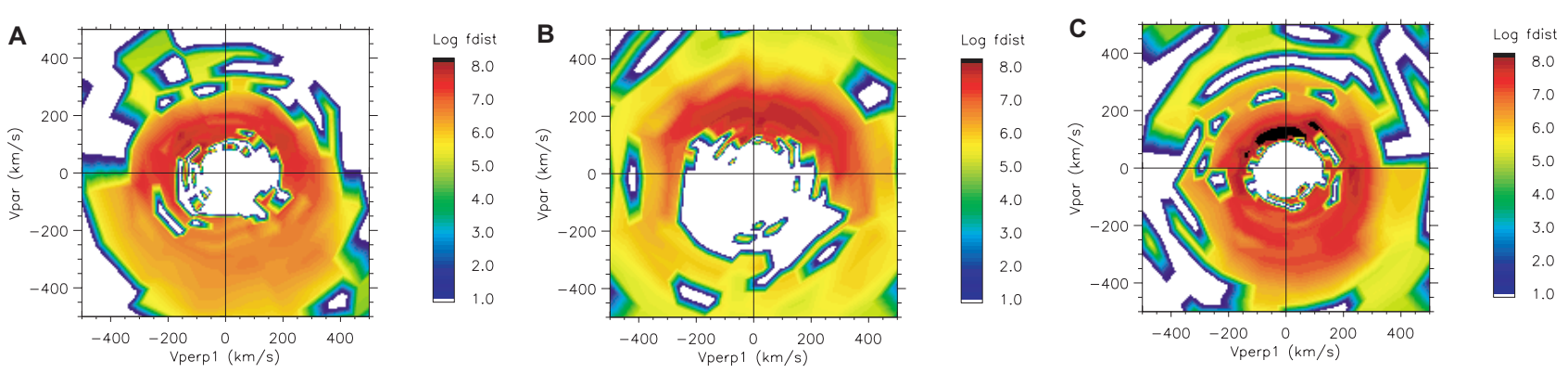

Fig. 7. Proton distribution functions from C4, showing shell-like features. (a) 02:54:59 UT, (b) 02:56:58 UT, (c) 03:09:58 UT. Shown is distribution function cuts in parallel and one perpendicular direction. Cuts of the distribution functions in perpendicular-perpendicular plane also display similar features (not shown).

$\mathrm{C} 1$ and $\mathrm{C} 4$. The points in phase-frequency space, panel (c), do not align along a line but are randomly distributed. The perpendicular separation of $\mathrm{C} 1$ and $\mathrm{C} 4$ is $\sim 60 \mathrm{~km}$, while the parallel separation is $\sim 40 \mathrm{~km}$. Thus, we observe no coherency for these emissions when the inter-spacecraft separation is smaller in parallel but longer in the perpendicular direction than for the pair C1-C2. Similarly low coherence is observed for all spacecraft pairs except the pair $\mathrm{C} 1-\mathrm{C} 2$, for all other times, for this boundary crossing. In summary, good coherency is only observed when the spacecraft are aligned almost along the same field line, and measured phase velocities are almost one order of magnitude lower than the Alfvén velocity. Since the perpendicular separation of all pair of spacecraft is greater than for the pair $\mathrm{C} 1-\mathrm{C} 2$, even though the parallel separation could be smaller, we draw the following conclusions:

- The wave emissions around the proton gyrofrequency observed on all four spacecraft have a coherence length of at least $80 \mathrm{~km}\left(\gtrsim 4 \rho_{p}\right)$ in parallel direction, and at least $30 \mathrm{~km}$ but at most $60 \mathrm{~km}\left(1-3 \rho_{p}\right)$ in the perpendicular direction.

- Measured perpendicular phase velocities are of the order of $100 \mathrm{~km} / \mathrm{s}$, an order of magnitude lower than the Alfvén velocity.

- The phase velocity estimate gives an upper bound of the perpendicular wavelength $\lambda_{\perp} \lesssim 50-100 \mathrm{~km}$.

\subsection{Ion distribution functions}

In Fig. 7 we show the ion distribution functions from $\mathrm{C} 4$ for three different time intervals. All panels show cuts in the parallel-perpendicular plane. Panels (a) and (b) are distribution functions from two different times in the HLBL. Panel (b) clearly shows the proton injection around 02:58 UT that is mentioned in Sect. 2.1. The distribution functions resembles a horse-shoe shape. Such distribution functions, formed due to mirroring of downgoing ions in the converging magnetic field, is expected to be present on recently reconnected field lines. Panel (c) shows an example of a distribution function from the cusp proper. It can be seen that the distribution function is more symmetric than in panels (a) and (b). This is consistent with these field lines being reconnected for a longer time so that the injected plasma has had time to mirror and thus form a more symmetric distribution function. In the plasma frame (approximately equal to the ion center of mass reference frame) the distributions have a positive gradient $\partial f / \partial v_{\|}, \partial f / \partial v_{\perp}>0$, for velocities around $100-200 \mathrm{~km} / \mathrm{s}$. This is close to the estimated phase velocity of the waves and indicates that ions can be a free energy source of the waves as speculated below in the Discussion section.

\section{Discussion}

\subsection{Wave properties}

Our objective is to interpret the above described observations using known theoretical concepts. To this end we initially analyze the observational facts in terms of linear waves in a homogeneous plasma. Later, we give arguments as to why the theoretical model must be extended to include nonlinear and inhomogeneous effects.

In Sect. 2.4 we found that the $\delta E_{\perp} / \delta B_{\perp}$-ratio is of the order of the local Alfvén velocity in the low frequency limit. Together with the fact that we observe wave emissions both below and above the local $f_{c p}$ suggests that these waves can be ion-whistlers (other names for this wave mode are compressional Alfvén waves or magnetosonic waves), which can exist both below and above $f_{c p}$. We performed a preliminary analysis of the wave properties using the Wave Distribution Function (WDF) technique (Oscarsson, 1994), that is based on a homogeneous plasma model. Parameters of the model were taken to correspond to real particle observations and real field parameters observed. We found that one can fit the data but only if we used rather large adjustments of the model parameters.

To give a better description of the waves we investigated what information single- and multiple-spacecraft measurements, such as polarization analysis and cross-correlation analysis, could provide (Sects. 2.4 and 2.5). 
From the multi-spacecraft cross-spectral analysis (Sect. 2.5) we concluded that the phase velocity projected on the C1-C2 separation axis should be lower than $\sim 300 \mathrm{~km} / \mathrm{s}$ and we argued that the most probable wave vector is almost perpendicular with respect to the ambient magnetic field $\left(k_{\perp} \gg k_{\|}\right)$, in which case the perpendicular phase velocity would be even lower, $v_{p h, \perp} \lesssim 100 \mathrm{~km} / \mathrm{s}$. This is an order of magnitude lower than the Alfvén speed obtained from background parameters, $v_{A} \sim 1300-1500 \mathrm{~km} / \mathrm{s}$. The phase velocity also gave an upper bound estimate of the perpendicular wavelength $\lambda_{\perp} \lesssim 50-100 \mathrm{~km}$.

The multi-spacecraft analysis reveals another feature of these waves. As shown in Sect. 2.5 the coherence length is at least $80 \mathrm{~km}$ in a direction parallel to the ambient magnetic field, and $30-60 \mathrm{~km}$ in the perpendicular direction. The relation between coherence length and wavelength is not trivial. However, as long as the wavelength is long compared to the interferometer axis, the coherency is large, even if the $\mathrm{k}$-spectrum is broad (see Kintner et al., 2000). Thus the low coherency in the perpendicular direction gives an upper bound estimate of the perpendicular length scale. The coherence length of $30-60 \mathrm{~km}$ corresponds to a perpendicular length scale of the order of one to three $\rho_{p}\left(\rho_{p} \sim 23 \mathrm{~km}\right)$, in agreement with the estimated characteristic wavelength above. This result is valid for frequencies both below and above $f_{c p}$.

No clear polarization could be found between the perpendicular wave components, e.g. right-hand polarization would be expected for ion-whistlers (e.g. Stix, 1992). However, a clear polarization signature was observed between the parallel component and the one perpendicular component. We noted that the ellipticity in this plane changes sign (sense of rotation) at the local $f_{c p}$. Such a change in ellipticity would be observed if for a given wave either $k_{\perp}$ or $k_{\|}$changed the sign. Note that in the perpendicular plane waves keep their polarization, e.g. whistlers are right-handed waves independent of propagation direction. In Sect. 2.5 we argued that indeed $k_{\|}$changes sign at $f_{c p}$ and thus this is consistent with the observed change of wave polarization in the plane spanned by parallel-perpendicular directions at the local $f_{c p}$ (Fig. 3). This also suggests that the waves below and above $f_{c p}$ should have very similar polarization properties, for example, by being the same plasma wave mode.

First we look at waves with frequencies below $f_{c p}$. The electron inertial length $c / \omega_{p e} \sim 3 \mathrm{~km}$ and the electron gyroradius $\rho_{e} \sim 0.3 \mathrm{~km}$ are both much less than the the ion-gyroradius $\rho_{p}$. Together with the fact that $m_{e} / m_{p} \ll \beta_{e} \sim 0.01-0.04 \ll 1$, this suggests that the waves below $f_{c p}$ can be identified as kinetic Alfvén waves (KAW). In a homogeneous plasma using thermal electrons, thermal ions and including finite frequency effects, the dispersion relation of KAW for frequencies below $f_{c p}$ is (e.g. Stasiewicz et al., 2000)

$$
\omega^{2}=k_{\|}^{2} v_{A}^{2}\left[1+k_{\perp}^{2}\left(\rho_{s}^{2}+\rho_{p}^{2}\right)-\frac{\omega^{2}}{\omega_{c p}^{2}}\left(1+k_{\perp}^{2} \rho_{p}^{2}\right)\right]
$$

where $\rho_{s}=\sqrt{\left(T_{e} / m_{p}\right)} / \omega_{c p}$.

In the frequency range above $f_{c p}$ the short characteristic perpendicular scale imposes certain constraints on possible wave modes. The perpendicular component for these waves should be of the order or even larger than the inverse iongyroradius $k_{\perp} \gtrsim \rho_{p}^{-1}$. This excludes ion-whistlers (compressional Alfvén waves) as the only present wave mode, since WHAMP (Rönnmark, 1982) calculations with the present plasma parameters show that the perpendicular wavelength for ion-whistlers is at least one order of magnitude longer than the observed coherence lengths. We cannot, however, exclude ion-whistlers altogether based on the length scale. A small part of the wave power could be due to whistler waves, but the dominant part should be due to another wave mode.

The measured perpendicular scale, together with the fact that these waves have a non-negligible magnetic field component, results in the identification of these waves as quasitransverse propagating, non-potential ion-cyclotron waves. There exist several ion-cyclotron modes for quasi-transverse propagation: quasi-longitudinal, ordinary and extra-ordinary. Another name for these modes could be electromagnetic ionBernstein modes, although the label ion-Bernstein is usually associated with the longitudinal (electrostatic) mode (see e.g. Akhiezer et al., 1975; Lominadze, 1981). It is worth noting the presence of peaks in the spectrum of the magnetic field near $f_{c p}$, suggesting the presence of Bernstein waves. The non-potential ordinary modes couple to the kinetic Alfvén waves around the cyclotron frequency for short perpendicular wavelengths, while the extra-ordinary mode couples to the ion-whistler wave (compressional Alfvén). The polarization analysis suggested that waves below and above $f_{c p}$ have the same polarization properties, which suggests that the relevant modes above $f_{c p}$ are the quasi-longitudinal and/or ordinary ion-cyclotron modes.

We have used the coherence lengths as a means of gaining information on scale lengths. In general, the short perpendicular coherence length makes wave-mode identification difficult. If good coherency is found between spacecraft for all spacecraft combinations, then a three-dimensional $\boldsymbol{k}$ can be determined, assuming a one-to-one correspondence between wave number and frequency. However, coherence lengths shorter than the spacecraft separation makes this impossible. No 3-D $\boldsymbol{k}$ can be determined with phase difference techniques due to bad coherency for some spacecraft pairs. For the same reason k-filtering cannot be applied.

Up to now we have considered linear waves in a homogeneous plasma. We present arguments why we probably must relax one or both of the constraints of linearity and homogeneity, in order to describe the waves with sufficient accuracy:

- Energetics: The ratio of energy fluxes in waves and ions is $\mathrm{PF} / \mathrm{IPF} \sim 2.5 \cdot 10^{-2}$ for waves with frequencies $f \sim f_{c p}$. This suggests that a significant part of the available energy is in the waves and that nonlinear effects can be important. 


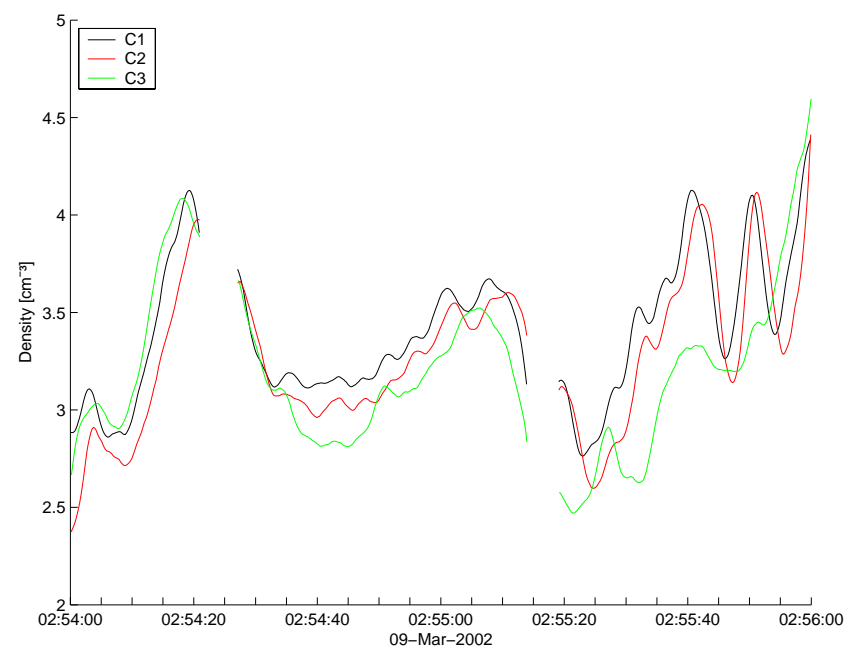

Fig. 8. Density estimates from EFW spacecraft potential measurements (see Pedersen et al., 2001, for a general description of the method). The data gaps are due to instrumental interference from WHISPER. For example, at 02:55:40 UT, C3 measures a different density compared to $\mathrm{C} 1$ and $\mathrm{C} 2$. This implies a local density gradient of $25 \%$ on the length scale of the spacecraft separation $\sim 50 \mathrm{~km}\left(2 \rho_{p}\right)$.

- Density gradients: Examining the density measured by different spacecraft at the same instant of time reveals that the plasma is inhomogeneous on the scale of the inter-spacecraft distances. The density can differ by as much as $25 \%$ over a distance of $\sim 50 \mathrm{~km}\left(2 \rho_{p}\right)$, see Fig. 8 .

Strong gradients on the scale of the ion-gyroradius, which is also the perpendicular wavelength, suggests that not only inhomogeneous but also nonlinear features must be taken into account. For example, for waves with frequencies below $f_{c p}$ this means that Eq. (4) must be extended to include nonlinearities and gradient (drift) effects. In this case nonlinear drift Alfvén waves can probably give a better description, as they are known to create field-aligned electromagnetic structures in the form of vortices which could explain the observed emissions, polarization and coherence lengths (see, e.g. Shukla et al., 1985; Chmyrev et al., 1988; Dubinin et al., 1988). To explore in more detail all these possibilities is a topic of our future work.

\subsection{Wave-particle interaction}

The Poynting flux along the ambient magnetic field as a function of frequency shows two important features (see, Sect. 2.3). First, below $f_{c p}$ the Poynting flux is predominantly downward (earthward) while it is mainly upward for frequencies above $f_{c p}$. This is consistent with a model where waves are generated with frequencies close to the local proton gyrofrequency in an extended region along the magnetic field line. If these waves propagate both upward and downward from the generation site, then the waves with frequencies below the local proton gyrofrequency are expected to come from above where their frequency comes close to the local proton gyrofrequency and vice versa for waves above the proton gyrofrequency. Secondly, the net Poynting flux for these waves is small in comparison to the Poynting flux carried by lower frequency waves, except in HLBL, where they are comparable. Thus, the waves with frequencies around the proton gyrofrequency cannot transport significant energy in the cusp while they still can be important for particle energization and energy redistribution through wave-particle and wave-wave interaction. Similar observations have been reported from the auroral zone (Chaston et al., 1998, 2002). In regions of auroral particle acceleration with upward current these studies reported electromagnetic waves at the proton cyclotron frequency and its harmonics, unstable to both electron as well as ion beams. The Poynting flux was found to be mainly upward above $f_{c p}$ and downward below, similar to our findings.

We observe a correspondence between the electromagnetic waves and the presence of protons (see, Sect. 2.2) The fact that the ion power flux is approximately 40 times larger than the Poynting flux for frequencies around $f_{c p}$, and that proton thermal velocities are of the order of wave phase velocities, makes it plausible that the protons are the source of free energy for the waves. Cluster also observes anisotropic electrons at the same time as the waves and protons. Preliminary analysis of electron power flux does not allow us to draw any conclusions about the possibility of electrons being the free energy source of waves; more care must be taken to reduce instrumental uncertainties. On the other hand, the electron distribution functions seem to indicate that electrons can be locally accelerated along the magnetic field lines to Alfvén speeds. This would be consistent with earlier studies (Hasegawa, 1976; Stasiewicz et al., 2000, and references therein) suggesting that anisotropic electron distribution functions can be due to acceleration by kinetic Alfvén waves. We leave the thorough analysis of electrons and the coupling between low-frequency Alfvén waves and high-frequency electron waves to our future study.

To identify the source of free energy we examined the proton distribution functions in Sect. 2.6. We found ion shell distributions with ion velocities at the location of the positive derivatives around $\sim 100-200 \mathrm{~km} / \mathrm{s}$, close to our estimates of the phase velocities of the waves. Ion shell distributions are known to generate, e.g. ion-Bernstein waves (Janhunen et al., 2003), while 2-D analogue ring distributions are known to generate, e.g. ion-whistlers (Perraut et al., 1982; Korth et al., 1984), and thus is a plausible source of the waves.

To investigate more quantitatively if the proton shell distributions can be the source of the waves we modeled the observed particle distributions in WHAMP. The proton shell was modeled by adding two Maxwellian distributions for the protons with characteristic parameters $\left(T_{p 1}=400 \mathrm{eV}\right.$, $\left.n_{p 1}=4 \mathrm{~cm}^{-3}\right)$ and $\left(T_{p 2}=130 \mathrm{eV}, n_{p 1}=-0.8 \mathrm{~cm}^{-3}\right)$. The second distribution has a negative density, in order to make the resulting distribution resemble a shell. The electrons were modeled by a single Maxwellian distribution with the 
parameters $\left(T_{e}=130 \mathrm{eV}, n_{p 1}=3.2 \mathrm{~cm}^{-3}\right)$. The output is presented in Fig. 9, which shows the first ion cyclotron harmonic for a value of $k_{\|}=0.1 \omega_{c p} / v_{p, t h}$. Panels (a) and (b) shows the real $(\omega)$ and imaginary parts $(\gamma)$ of the frequency, respectively, plotted as a function of $k_{\perp}$. We note that indeed considerable wave growth $\left(\gamma \sim 10^{-2} \omega_{c p}\right.$, with a maximum of $\gamma=0.08 f_{c p}=0.13 \mathrm{~s}^{-1}$ ) occurs for perpendicular wavelengths $\lambda_{\perp} \simeq 1.5 \rho_{p}$, where we have used $v_{p, t h} \sim 300 \mathrm{eV}$, $k_{\perp}=3 \omega_{c p} / v_{p, t h}$ and $f_{c p}=1.6 \mathrm{~Hz}$. This is in good agreement with the observed wavelengths obtained from the multispacecraft cross-spectral analysis above $\left(\lambda_{\perp} \sim 1-4 \rho_{p}\right)$. The $E_{\perp} / B_{\perp}$-ratio is plotted in panel (c), indicating the nonpotential (magnetic component) nature of the waves. This ratio is in agreement with Fig. 2. We finally note that since the electrons are Maxwellian, they do not contribute to the observed wave growth in our model. Modeling the distributions more carefully and investigating other wave modes than ion cyclotron waves is a topic we leave for a future study.

There are other possible free energy sources that we have not discussed in detail here. For example, one such source can be shear velocity flows in the plasma that are often observed in the cusp and its boundary layers and are also seen in our event. In addition, large-scale currents, such as diamagnetic currents, can be a source of free energy. The magnetic field spectrum shows a combination of a spectral peak around $f_{c p}$ and a broad-band spectra with different slopes below and above $f_{c p}$ (Fig. 2), which also indicate a possibility of having several wave sources. Kinked double slope spectra in the cusp has also been previously reported from Interball- 1 and Polar observations (Savin et al., 2002).

In summary, ion power flux, the Poynting flux distribution over frequency, peaks at $f_{c p}$ and its multiples, together with the proton shell distribution functions and sheared plasma flow, make it probable that the waves are generated locally in the cusp along the field lines, from one or possibly more sources by an instability which favours the ion-cyclotron frequency.

\section{Summary and conclusions}

We have done a detailed study of waves with frequencies near the proton gyrofrequency in the high-altitude cusp for northward IMF as observed by the Cluster spacecraft. Our main conclusions are:

- All four spacecraft observe enhanced emissions in the electric and magnetic fields both below and above the proton gyrofrequency. The emissions are most pronounced at the poleward edge of the cusp in localized regions of injected $300 \mathrm{eV}$ ions. The injected ions are most probably caused by the reconnection at the magnetopause tailward (higher latitude side) of the cusp as expected for the observed northward IMF.

- Single spacecraft measurements of the $\delta E_{\perp} / \delta B_{\perp}$-ratio show that the waves are electromagnetic with a
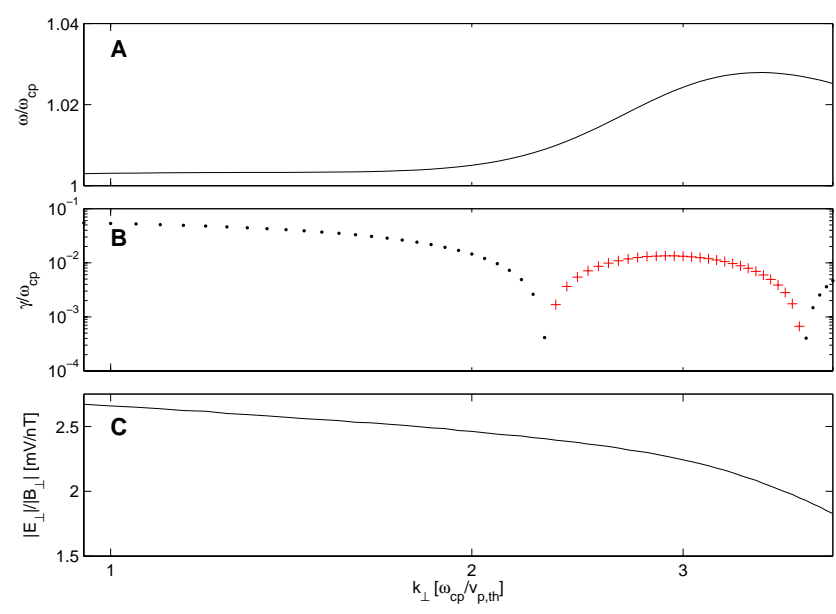

Fig. 9. Ion cyclotron mode obtained from WHAMP with proton shell and Maxwellian electron distributions modeled from particle measurements. (a) Frequency vs. perpendicular wavenumber. (b) Imaginary part of frequency vs. perpendicular wave number. Black dots stand for $\gamma<0$ (damping) and red crosses for $\gamma>0$ (growth). (c) $E_{\perp} / B_{\perp}$-ratio. The parallel wave number was $k_{\|}=0.1 \omega_{c p} / v_{p, t h}$.

$\delta E_{\perp} / \delta B_{\perp}$-ratio of the order of the local Alfvén speed in the low frequency limit.

- There is a low degree of polarization in the plane perpendicular to the ambient magnetic field, due to a change in the sense of rotation on a time scale comparable to the wave period. However, there is a clear polarization in one parallel-perpendicular plane. A change in the sense of polarization in the parallel-perpendicular plane is consistent with a change in the sign of $k_{\|}$with respect to $f_{c p}$.

- The measurements are consistent with $k_{\perp} \gg k_{\|}$. Crossspectral analysis of the wave magnetic field between spacecraft results in an estimate of the perpendicular phase velocity of the order of $100 \mathrm{~km} / \mathrm{s}$, which is much less than the local Alfvén speed $v_{A} \sim 1300-1500 \mathrm{~km} / \mathrm{s}$. From the phase velocity follows that the perpendicular wavelength should be a few proton gyroradius or less, $\lambda_{\perp} \lesssim 2-4 \rho_{p}$.

- The waves have a coherence length which is of the order of one ion-gyroradius in the direction perpendicular to the ambient magnetic field and a few times larger or more in the parallel direction.

- The coherence length gives an upper bound for a perpendicular wavelength or length scale of the order of the ion-gyroradius, agreeing in order with the estimated wavelength. This gives constraints on possible wave modes and implies that kinetic (finite gyroradius) effects are important.

- Below $f_{c p}$ the waves supposedly belong to the kinetic Alfvén branch. Above $f_{c p}$ non-potential ion-cyclotron 
waves are the most probable wave mode which can couple to the kinetic Alfvén wave around the cyclotron frequency for short perpendicular wavelengths.

- Large plasma density gradients are observed on the scale of perpendicular wavelengths (on the order of the ion-gyroradius), which suggest that nonlinear and nonhomogeneous effects are important.

- The Poynting flux projected on the ambient magnetic field is mainly downward (earthward) below the local $f_{c p}$, and upward above the local $f_{c p}$. This feature of the Poynting flux is consistent with the waves being generated near the local proton gyrofrequency in an extended region along the flux tube.

- A likely source of free energy driving the waves with frequencies around $f_{c p}$ are injected protons. First, the observed waves show a correspondence to the proton flux. Secondly, the calculated ion power flux is approximately 40 times larger than the wave Poynting flux. Finally, the injected protons show shell-like distribution functions that have positive gradients and are thus kinetically unstable; the velocity at the positive gradients is of the order of the measured phase velocity of waves. Linear wave growth calculations using WHAMP supports this picture by showing significant wave growth at perpendicular wavelengths agreeing with measurements.

Here we have shown that multipoint measurements are essential for studying waves in the cusp and its boundaries. In our future work we believe that it is important to look at wave models extended to include either nonlinearities and/or inhomogeneities on scales of the order of the ion-gyroradius, as well as velocity shear effects.

Acknowledgements. D. Sundkvist and V. Krasnoselskikh acknowledge financial support from the European Community's Human Potential Programme under contract HPRN-CT-2001-00314 "Turbulent Boundary Layers in Geospace Plasmas".

D. Sundkvist also wish to thank GradU, Uppsala University, for financial support, and A. Tjulin for useful discussions.

A. Vaivads research is supported by the Swedish Research Council.

Topical Editor T. Pulkkinen thanks J. Blecki and C. Chaston for their help in evaluating this paper.

\section{References}

Akhiezer, A. I., Akhiezer, I. A., Polovin, R. V., Sitenko, A. G., and Stepanov, K. N.: Plasma Electrodynamics, Volume 1: Linear Theory, Pergamon Press, 1975.

André, M.: Waves and wave-particle interactions in the auroral region, J. Atmos. Terr. Phys., 59, 1687-1712, 1997.

André, M., Koskinen, H., Gustafsson, G., and Lundin, R.: Ion waves and upgoing ion beams observed by the Viking satellite, Geophys. Res. Lett., 14, 463-466, 1987.

André, M., Norqvist, P., Andersson, L., Eliasson, L., Eriksson, A. I., Blomberg, L., Erlandson, R. E., and Waldemark, J.: Ion energization mechanisms at $1700 \mathrm{~km}$ in the auroral region, J. Geophys. Res., 103, 4199-4222, 1998.
Balogh, A., Dunlop, M. W., Cowley, S. W. H., Southwood, D. J., Thomlinson, J. G., Glassmeier, K. H., Musmann, G., Luhr, H., Buchert, S., Acuña, M. H., Fairfield, D. H., Slavin, J. A., Riedler, W., Schwingenschuh, K., and Kivelson, M. G.: The Cluster Magnetic Field Investigation, Space Sci. Rev., 79, 65-91, 1997.

Bouhram, M., Malingre, M., Jasperse, J. R., and Dubouloz, N.: Modeling transverse heating and outflow of ionospheric ions from the dayside cusp/cleft. 1 A parametric study, Ann. Geophys., 21, 1753-1771, 2003a,

SRef-ID: 1432-0576/ag/2003-21-1753.

Bouhram, M., Malingre, M., Jasperse, J. R., Dubouloz, N., and Sauvaud, J.-A.: Modeling transverse heating and outflow of ionospheric ions from the dayside cusp/cleft. 2 Applications, Ann. Geophys., 21, 1773-1791, 2003b,

SRef-ID: 1432-0576/ag/2003-21-1773.

Carozzi, T. D., Thidé, B., Leyser, T. B., Komrakov, G., Frolov, V., Grach, S., and Sergeev, E.: Full polarimetry measurements of stimulated electromagnetic emissions: First results, J. Geophys. Res., 21 395-21 408, 2001.

Chaston, C. C., Ergun, R. E., Delory, G. T., Peria, W., Temerin, M., Cattell, C., Strangeway, R., McFadden, J. P., Carlson, C. W., Elphic, R. C., Klumpar, D. M., Peterson, W. K., Moebius, E., and Pfaff, R.: Characteristics of electromagnetic proton cyclotron waves along auroral field lines observed by FAST in regions of upward current, Geophys. Res. Lett., 25, 2057-2060, 1998.

Chaston, C. C., Bonnell, J. W., McFadden, J. P., Ergun, R. E., and Carlson, C. W.: Electromagnetic ion cyclotron waves at proton cyclotron harmonics, J. Geophys. Res., 107, 8-1, 2002.

Chmyrev, V. M., Bilichenko, S. V., Pokhotelov, O. A., Marchenko, V. A., Lazarev, V. I., Streltsov, A. V., and Stenflo, L.: Alfvén Vorticies and Related Phenomena in the Ionosphere and the Magnetosphere, Physica Scripta, 38, 841-854, 1988.

Cornilleau-Wehrlin, N., Chauveau, P., Louis, S., Meyer, A., Nappa, J. M., Perraut, S., Rezeau, L., Robert, P., Roux, A., de Villedary, C., de Conchy, Y., Friel, L., Harvey, C. C., Hubert, D., Lacombe, C., Manning, R., Wouters, F., Lefeuvre, F., Parrot, M., Pincon, J. L., Poirier, B., Kofman, W., and Louarn, P.: The Cluster Spatio-Temporal Analysis of Field Fluctuations (STAFF) Experiment, Space Sci. Rev., 79, 107-136, 1997.

Dubinin, E. M., Volokitin, A. S., Israelevich, P. L., and Nikolaeva, N. S.: Auroral electromagnetic disturbances at altitudes of 900km: Alfvén wave turbulence, Planet. Space Sci., 36, 949962, 1988.

Erlandson, R. E. and Zanetti, L. J.: A statistical study of auroral electromagnetic ion cyclotron waves, J. Geophys. Res., 103, 4627-4636, 1998

Fredricks, R. W. and Russell, C. T.: Ion cyclotron waves observed in the polar cusp, J. Geophys. Res., 78, 2917, 1973.

Gurnett, D. A.: Auroral Plasma Waves, in Auroral Physics, edited by: Meng, C.-I., Rycroft, M. J., and Frank, L. A., Cambrige University Press, Cambridge, UK, 241-245, 1991.

Gurnett, D. A. and Frank, L. A.: A region of intense plasma wave turbulence on auroral field lines, J. Geophys. Res., 82, 10311050, 1977.

Gustafsson, G., André, M., Matson, L., and Koskinen, H.: On waves below the local proton gyrofrequency in auroral acceleration regions, J. Geophys. Res., 95, 5889-5904, 1990.

Gustafsson, G., Boström, R., Holback, B., Holmgren, G., Lundgren, A., Stasiewicz, K., Åhlén, L., Mozer, F. S., Pankow, D., Harvey, P., Berg, P., Ulrich, R., Pedersen, A., Schmidt, R., Butler, A., Fransen, A. W. C., Klinge, D., Thomsen, M., Fälthammar, C.-G., Lindqvist, P.-A., Christenson, S., Holtet, J., Lybekk, B., 
Sten, T. A., Tanskanen, P., Lappalainen, K., and Wygant, J.: The Electric Field and Wave Experiment for the Cluster Mission, Space Sci. Rev., 79, 137-156, 1997.

Hamrin, M., Norqvist, P., Hellström, T., André, M., and Eriksson, A. I.: A statistical study of ion energization at $1700 \mathrm{~km}$ in the auroral region, Ann. Geophys., 20, 1943-1958, 2002,

\section{SRef-ID: 1432-0576/ag/2002-20-1943.}

Hasegawa, A.: Particle acceleration by MHD surface wave and formation of aurora, J. Geophys. Res., 81, 5083-5090, 1976.

Janhunen, P., Olsson, A., Vaivads, A., and Peterson, W. K.: Generation of Bernstein waves by ion shell distributions in the auroral region, Ann. Geophys., 21, 881-891, 2003,

SRef-ID: 1432-0576/ag/2003-21-881.

Johnstone, A. D., Alsop, C., Burge, S., Carter, P. J., Coates, A. J., Coker, A. J., Fazakerley, A. N., Grande, M., Gowen, R. A., Gurgiolo, C., Hancock, B. K., Narheim, B., Preece, A., Sheather, P. H., Winningham, J. D., and Woodliffe, R. D.: Peace: a Plasma Electron and Current Experiment, Space Sci. Rev., 79, 351-398, 1997.

Kaufmann, R. L. and Kintner, P. M.: Upgoing ion beams, I - Microscopic analysis, J. Geophys. Res., 87, 10 487-10 502, 1982.

Kintner, P. M., Franz, J., Schuck, P., and Klatt, E.: Interferometric coherency determination of wavelength or what are broadband ELF waves?, J. Geophys. Res., 105, 21 237-21 250, 2000.

Korth, A., Kremser, G., Perraut, S., and Roux, A.: Interaction of particles with ion cyclotron waves and magnetosonic waves. Observations from GEOS 1 and GEOS 2, Planet. Space Sci., 32, 1393-1406, 1984.

Labelle, J. and Kintner, P. M.: The measurement of wavelength in space plasmas, Rev. Geophys., 27, 495-518, 1989.

Le, G., Blanco-Cano, X., Russell, C. T., Zhou, X.-W., Mozer, F., Trattner, K. J., Fuselier, S. A., and Anderson, B. J.: Electromagnetic ion cyclotron waves in the high altitude cusp: Polar observations, J. Geophys. Res., 106, 19067-19079, 2001.

Lominadze, D. G.: Cyclotron Waves in Plasma, Pergamon Press, 1981.

Nykyri, K., Cargill, P. J., Lucek, E. A., Horbury, T. S., Balogh, A., Lavraud, B., Dandouras, I., and Rème, H.: Ion cyclotron waves in the high altitude cusp: CLUSTER observations at varying spacecraft separations, Geophys. Res. Lett., 30, 12-1, 2003.

Nykyri, K., Cargill, P. J., Lucek, E., Horbury, T., Lavraud, B., Balogh, A., Dunlop, M. W., Bogdanova, Y., Fazakerley, A., Dandouras, I., and Rème, H.: Cluster observations of magnetic field fluctuations in the high-altitude cusp, Ann. Geophys., 22, 24132429, 2004,

\section{SRef-ID: 1432-0576/ag/2004-22-2413.}

Oscarsson, T.: Dual principles in maximum entropy reconstruction of the wave distribution function, J. Comput. Ph., 110, 221-233, 1994.

Pedersen, A., Décréau, P., Escoubet, C.-P., Gustafsson, G., Laakso, H., Lindqvist, P.-A., Lybekk, B., Masson, A., Mozer, F., and Vaivads, A.: Four-point high time resolution information on electron densities by the electric field experiments (EFW) on Cluster, Ann. Geophys., 19, 1483-1489, 2001,

SRef-ID: 1432-0576/ag/2001-19-1483

Perraut, S., Roux, A., Robert, P., Gendrin, R., Sauvaud, J.-A., Bosqued, J.-M., Kremser, G., and Korth, A.: A systematic study of ULF waves above F/H plus/ from GEOS 1 and 2 measurements and their relationships with proton ring distributions, J. Geophys. Res., 87, 6219-6236, 1982.
Rème, H., Bosqued, J. M., Sauvaud, J. A., Cros, A., Dandouras, J., Aoustin, C., Bouyssou, J., Camus, T., Cuvilo, J., Martz, C., Medale, J. L., Perrier, H., Romefort, D., Rouzaud, J., D`Uston, C., Mobius, E., Crocker, K., Granoff, M., Kistler, L. M., Popecki, M., Hovestadt, D., Klecker, B., Paschmann, G., Scholer, M., Carlson, C. W., Curtis, D. W., Lin, R. P., McFadden, J. P., Formisano, V., Amata, E., Bavassano-Cattaneo, M. B., Baldetti, P., Belluci, G., Bruno, R., Chionchio, G., di Lellis, A., Shelley, E. G., Ghielmetti, A. G., Lennartsson, W., Korth, A., Rosenbauer, H., Lundin, R., Olsen, S., Parks, G. K., McCarthy, M., and Balsiger, H.: The Cluster Ion Spectrometry (CIS) Experiment, Space Sci. Rev., 79, 303-350, 1997.

Rönnmark, K.: Waves in homogeneous, anisotropic multicomponent plasmas (WHAMP), Tech. rep., KRI, 1982.

Russel, C. T., Chappell, C. R., Montgomery, M. D., Neugebauer, M., and Scarf, F. L.: Ogo 5 observations of the polar cusp on November 1, 1968, J. Geophys. Res., 76, 6743, 1971.

Santolík, O., Pickett, J. S., Gurnett, D. A., and Storey, L. R. O.: Magnetic component of narrowband ion cyclotron waves in the auroral zone, J. Geophys. Res., 107, 17-1, 2002.

Savin, S., Zelenyi, L., Maynard, N., Sandahl, I., Kawano, H., Russell, C. T., Romanov, S., Amata, E., Avanov, L., Blecki, J., Buechner, J., Consolini, G., Gustafsson, G., Klimov, S., Marcucci, F., Nemecek, Z., Nikutowski, B., Pickett, J., Rauch, J. L., Safrankova, J., Skalsky, A., Smirnov, V., Stasiewicz, K., Song, P., Trotignon, J. G., and Yermolaev, Y.: Multi-spacecraft tracing of turbulent boundary layer, Adv. Space Res., 30, 2821-2830, 2002.

Savin, S., Zelenyi, L., Romanov, S., Sandahl, I., Pickett, J., Amata, E., Avanov, L., Blecki, J., Budnik, E., Büchner, J., Cattell, C., Consolini, G., Fedder, J., Fuselier, S., Kawano, H., Klimov, S., Korepanov, V., Lagoutte, D., Marcucci, F., Mogilevsky, M., Nemecek, Z., Nikutowski, B., Nozdrachev, M., Parrot, M., Rauch, J., Romanov, V., Romantsova, T., Russell, C., Safrankova, J., Sauvaud, J., Skalsky, A., Smirnov, V., Stasiewicz, K., Trotignon, J., and Yermolaev, Y.: Magnetosheath-cusp interface, Ann. Geophys., 22, 183-212, 2004,

SRef-ID: 1432-0576/ag/2004-22-183.

Scarf, F. L., Fredricks, R. W., Green, I. M., and Russell, C. T.: Plasma waves in the dayside polar cusp, 1, Magnetospheric observations, J. Geophys. Res., 77, 2274, 1972.

Shukla, P. K., Yu, M. Y., and Varma, R. K.: Formation of kinetic Alfvén vortices, Phys. Lett. A, 109, 322-324, 1985.

Stasiewicz, K., Bellan, P., Chaston, C., Kletzing, C., Lysak, R., Maggs, J., Pokhotelov, O., Seyler, C., Shukla, P., Stenflo, L., Streltsov, A., and Wahlund, J.-E.: Small Scale Alfvénic Structure in the Aurora, Space Sci. Rev., 92, 423-533, 2000.

Stenberg, G., Oscarsson, T., André, M., and Chaston, C. C.: Investigating wave data from the FAST satellite by reconstructing the wave distribution function, J. Geophys. Res., 107, 19-1, 2002.

Stix, T. H.: Waves in Plasmas, Am. Inst. of Phys., New York, 1992.

Temerin, M. and Lysak, R. L.: Electromagnetic ion cyclotron mode (ELF) waves generated by auroral electron precipitation, J. Geophys. Res., 89, 2849-2859, 1984.

Wahlund, J.-E., Yilmaz, A., Backrud, M., Sundkvist, D., Vaivads, A., Winningham, D., André, M., Balogh, A., Bonnell, J., Buchert, S., Carozzi, T., Cornilleau, N., Dunlop, M., Eriksson, A. I., Fazakerley, A., Gustafsson, G., Parrot, M., Robert, P., and Tjulin, A.: Observations of auroral broadband emissions by CLUSTER, Geophys. Res. Lett., 30, 17-1, 2003. 